\title{
AS METODOLOGIAS ATIVAS COMO ESTRATÉGIAS PARA O DESENVOLVIMENTO DE COMPETÊNCIAS DE INTELIGÊNCIA EMOCIONAL NOS ESTUDANTES DO ENSINO SUPERIOR
}

\author{
Susana Sá ${ }^{1}$, José Morais² e Fernando Almeida ${ }^{3}$ \\ 1'Universidade Lusófona do Porto, CeiED, Porto, Portugal. susanaemiliasa@gmail.com; \\ ${ }^{2}$ ISPGAYA, CEOS.PP, Porto, Portugal. jmorais@ispgaya.pt; \\ ${ }^{3}$ ISPGAYA, INESC TEC, Porto, Portugal. falmeida@ispgaya.pt.
}

\begin{abstract}
Resumo. As metodologias ativas são pontos de partida para avanços nos processos de reflexão, de integração cognitiva, de generalização, de reelaboração de novas práticas, de modo a que o Professor consinta ao estudante do Ensino Superior desenvolver cognitivamente a Inteligência Emocional. Este trabalho, de natureza qualitativa, tem como objetivo apresentar um diagnóstico de indicadores de aprendizagem de Inteligência Emocional, no sentido de responder à seguinte questão de pesquisa: quais as estratégias de aprendizagem para desenvolvimento de competências de IE nos estudantes do Ensino Superior? Realizaram-se dois focus group junto de duas instituições de Ensino Superior Público e Privado, nos quais participaram 10 estudantes e 4 professores. O conteúdo das entrevistas foi analisado com o software de análise qualitativa webQDA®. Identificaram-se e analisaram-se as seguintes categorias: autoconhecimento, auto-regulação, motivação, empatia, skills sociais e aprendizagem. Podemos concluir que as metodologias ativas, Problem Based Learning e Inverted Classroom, poderão contribuir para desenvolver skills de IE em estudantes de ensino Superior.
\end{abstract}

Palavras-chave: Ensino Superior; Inteligência Emocional; Metodologias Ativas; webQDA®.

\section{THE ACTIVE METHODOLOGIES AS STRATEGIES FOR DEVELOPMENT EMOTIONAL} INTELLIGENCE SKILLS IN HIGHER EDUCATION STUDENTS

\begin{abstract}
Active methodologies are starting points for advances in the processes of reflection, cognitive integration, generalization, and re-elaboration of new practices, so that the Teacher consents to the Higher Education student to develop Emotional Intelligence cognitively. This qualitative study aims to present a diagnosis of Emotional Intelligence learning indicators, in order to answer the following research question: what are the learning strategies for developing Emotional Intelligence competences in Higher Education students? Two focus groups were held with two institutions of Public and Private Higher Education, in which 10 students and 4 Teachers participated. The content of the interviews was analyzed using the webQDA® qualitative analysis software. The following categories were identified and analyzed: self-knowledge, self-regulation, motivation, empathy, social skills and learning. We can conclude that the active methodologies, Problem Based Learning and Inverted Classroom, can contribute to develop Emotional Intelligence skills in Higher Education students.
\end{abstract}

Keywords: Active Methodologies; Emotional Intelligence; High Education; webQDA®.

\section{INTRODUÇÃO}

O desenvolvimento de competências no âmbito da Inteligência Emocional (IE), e mais concretamente no âmbito do Ensino Superior, deverá afigurar-se como uma preocupação premente. Efetivamente, poderemos constatar que a IE se mantém como skill solicitada no mundo do trabalho, prevendo-se a continuidade futura desta solicitação, apesar de algumas competências anteriormente solicitadas perderem importância no contexto do desenvolvimento tecnológico aplicado (Câmpeanu-Sonea, Sonea, \& Bresfelean, 2013). 
Entendemos a IE como o conjunto de processos mentais que permitem ao indivíduo reconhecer, usar, compreender e gerir as emoções ou os estados emocionais, de si próprio e dos outros, auxiliando-o nos pensamentos e nos comportamentos (Brakett \& Salovey, 2006), ou seja, processos que remetem para competências intra e interpessoais (Mayer, Salovey, \& Caruso, 2004), que podem e devem ser trabalhadas e desenvolvidas nos indivíduos no contexto do Ensino Superior. A esta noção de IE, acrescentamos que corresponde a um conjunto de habilidades mentais que permitem o raciocínio e a resolução de problemas a partir da perceção e conhecimento de padrões das emoções (Cherniss, Extein, Goleman, \& Weissberg, 2006; Franco, 2007; Mayer, Caruso, \& Salovey, 2000).

O Ensino Superior deve assumir o desenvolvimento de competências de IE, bem como a sensibilização dos seus estudantes para a relevância destas competências, como uma prioridade, desenvolvendo e aplicando recursos educativos no sentido de proporcionar contextos facilitadores do desenvolvimento da capacidade dos indivíduos para processar informação emocional, capacidade esta que interfere diretamente com o desempenho do indivíduo entendido de forma ampla, ou seja, interfere ou relaciona-se com as suas outras competências, potenciando-as ou diminuindo-as.

No fundo, a IE reporta-se à capacidade dos indivíduos para processar toda a informação de cariz emocional (Brakett \& Salovey, 2006). Goleman (1995), sugere que a IE é mais importante do que o QI para predizer o sucesso na vida, incluindo o sucesso académico, pelo que a consideração do papel das emoções no sucesso académico e no contexto educacional tem ganho relevância (Sousa \& Dias, 2011), procurando-se estudar a relação entre o rendimento académico e a El dos indivíduos. Assim, apesar de as Instituições de Ensino Superior oferecerem experiências de "ensino virtual", "salas de aula virtuais", etc., isto só por si não é suficiente, uma vez que estas Instituições devem promover experiências inovadoras nos processos de ensino-aprendizagem, usando as TIC e contando com mudanças nas estratégias de ensino dos Professores, sistemas de comunicação Professor-estudante e o repensar a utilização dos materiais de aprendizagem.

\subsection{A Inteligência Emocional e o FLYGBY}

Entende-se o conceito de IE enquadrado nas chamadas inteligências pessoais, de que são exemplos a interpessoal e a intrapessoal (Almeida, 2019). Assumindo-se como uma forma de inteligência geral distinta da inteligência cognitiva, "a IE reflete o potencial de um indivíduo no 
comportamento adaptativo inteligente e promove o sucesso pessoal, a felicidade e o bemestar geral" (Sousa \& Dias, 2011, p. 96).

Os autores Mayer e Salovey (1997) conceptualizam a IE como um conjunto de habilidades mentais que permitem perceber e conhecer o significado de padrões das emoções, e raciocinar e resolver problemas a partir deles. O constructo de IE é caraterizado por um conjunto de processos mentais que permitem ao indivíduo reconhecer, usar, compreender e gerir as emoções ou os estados emocionais, quer de si próprio quer dos outros, de modo a auxiliar os pensamentos e os comportamentos (Cherniss, Extein, Goleman, \& Weissberg, 2006). Nas palavras dos autores, a IE refere-se às capacidades de: "perceber, avaliar e expressar as emoções, de aceder e de gerar sentimentos que facilitem o pensamento, de compreender e analisar informação emocional e de usar o conhecimento emocional, de regular as emoções para promover o crescimento emocional e intelectual" (Mayer \& Salovey, 1997, p. 15).

Um exemplo destes recursos são os Jogos Sérios, como o FLIGBY (Almeida, 2019; Almeida \& Buzády 2019), que não sendo imersivos, permitem o contato, de forma mais ou menos explicita, dos estudantes com o conceito, dimensões e indicadores de inteligência emocional, mas também o desenvolvimento e aplicação de skills ligadas diretamente ao conceito de IE, constituindo-se em aprendizagens a aplicar e rentabilizar no sentido do sucesso escolar e também do sucesso profissional.

Além de apresentarmos o conceito de IE, numa visão holística dentro de vários aportes, tentamos explorar o entendimento do conceito no âmbito de um jogo sério (FLIGBY), associado à teoria do Flow e à Psicologia Positiva (Almeida \& Buzádi, 2019), partindo do pressuposto que a IE está associada à performance, sendo um construto humano alterável ao longo do tempo, ou seja, plausível de desenvolvimento em cada indivíduo, desde que investido.

Os estados de Flow do FLIGBY podem ser descritos em termos das seguintes condições e características básicas (Csikszentmihalyi, 2003, pp. 42-56): a) Equilíbrio entre desafios e habilidades; b) Metas claras; c) Feedback imediato e claro (não precisa ser positivo, mas deve ser construtivo); d) Concentração intensa; e) Ação sem esforço; f) Perda do ego; g) Sentido de controle; g) Distorção da experiência temporal (inconsciente do tempo, espaço, ruído, fome); e h) Realizar uma atividade porque "se sente bem", não na expetativa de qualquer recompensa externa. 
Vários autores exploraram os benefícios oferecidos pela teoria de Flow em várias atividades. De um modo geral, esses benefícios podem ser agrupados em três dimensões (Alexiou et al., 2012; Almeida \& Buzády, 2019; Sahoo \& Sahu, 2009; Zumeta et al., 2016): a) Aumentar o repertório emocional, cognitivo e social do indivíduo; b) Aumentar o bem-estar e o desempenho dos funcionários; c) Aumentar a motivação, o sentido de comprometimento; e d) as perceções de crescimento pessoal.

Assim, afirmamos o ensino, e concretamente o Ensino Superior, como um contexto onde as skills relacionadas à IE devem ser obrigatoriamente trabalhadas, apresentando-se os jogos sérios como recurso educativo privilegiado para a prossecução deste objetivo.

Os autores Buzády e Almeida (2019) referem-se à IE como a capacidade de (e de uma forma positiva) identificar, compreender, reconhecer e gerenciar emoções, as nossas emoções e as emoções de outros personagens do jogo FLIGBY.

Acrescentam, portanto, algo mais ao conceito (resumido) de IE apresentado como indicador de performance no jogo, aproximando-se de uma abordagem mais elaborada deste conceito. Os autores realizam uma apologia do jogo sério como uma solução não imersiva para o desenvolvimento de skills relacionadas com a IE em estudantes do Ensino Superior, que efetivamente, podem até nem estar familiarizados com o conceito. $\mathrm{O}$ jogo apresenta-se como uma oportunidade de contato com o tema e também como a base de desenvolvimento de uma metodologia de ensino aprendizagem não convencional que pode ser usada em moldes mais latos no sentido de desenvolvimento de competências de IE na educação, dando azo a aplicações não só em unidades curriculares ligadas ao empreendedorismo, mas também no âmbito de outros domínios disciplinares. Basicamente, trata-se de métodos avançados de ensino e aprendizagem, denominados, Game-Based Learning (GBL), que se centram no design, desenvolvimento e aplicação de jogos na educação (Almeida, 2019).

\subsection{Metodologias ativas}

Entende-se que as metodologias ativas são pontos de partida para avanços nos processos de reflexão, de integração cognitiva, de generalização, de reelaboração de novas práticas, de modo a que o Professor consinta ao estudante desenvolver-se cognitivamente (Leite et al., 2018). É essencial uma renovação da formação profissional do Professor das Instituições do Ensino Superior, que possibilite a reconfiguração dos saberes e das práticas pedagógicas, ultrapassando a simples transmissão de conhecimentos, e envolva o estudante na 
assimilação e aplicação a novas situações do conhecimento curricular, tornando-o agente do seu desenvolvimento pessoal e profissional (Xavier, \& Leite, 2019). Os autores Ó, Almeida, Viana, Sanches e Paz (2019) equacionam as metodologias no sentido de os Professores possuírem performance elevada em contexto de sala de aula.

Assim, urge surgir, nas Instituições de Ensino Superior, novas metodologias de ensino, que: a) evidenciem princípios de autonomia; b) desenvolvam estratégias de ensino baseadas em pedagogias reflexivas e críticas; c) promovam relações abertas e informais, entre Professor e estudante, em que a comunicação se torna multilateral (Berbel, 2011). Algumas propostas de metodologias para o Ensino Superior são o Estudo Baseado em Problemas - PBL (Problem Based Learning) e a Sala de Aula Invertida (Inverted Classroom ou Flipped Classroom).

O PBL é uma metodologia de ensino (Ribeiro, 2008; Sá, Alves, \& Costa, 2016), cuja génese vem das teorias construtivistas da aprendizagem. Nesta metodologia, o docente permite que o estudante estabeleça o seu próprio conhecimento, desenvolvendo uma aprendizagem autónoma tendo como base processos de aprendizagem ativa, recorrendo a uma avaliação diferenciada, permitindo a apropriação de conhecimentos metacognitivos (Sá \& Costa-Lobo, 2019). Esta apropriação é apoiada por Sá, Alves e Costa (2016) que referem que o próprio conhecimento terá de ser visto pelos estudantes sob um ponto de vista mais ativo ou proactivo, autónomo ou colaborativo.

As Inverted Classroom (Erwen, Wenming, \& Chengxing, 2018; Rossi, 2014), têm como objetivo dinamizar, em dois estágios, os métodos que apresentam resultados significativos para a aprendizagem. No início, os estudantes recolhem informações para depois as assimilarem, sempre com a mediação do Professor, que tem como missão ajudar os estudantes a adquirirem mais responsabilidade e uma autonomia progressiva, assumindo o papel de facilitador do processo de aprendizagem (Erwen, Wenming, \& Chengxing, 2018; Fidalgo-Blanco, Sein-Echaluce, \& Garcia-Peñalvo, 2020; Rossi, 2014).

O papel do Ensino Superior tem como objetivo desenvolver a capacidade do estudante para a resolução de problemas, explorar e desenvolver a argumentação, a discussão, a suposição, desenvolvendo capacidades de IE para o sucesso académico e profissional. 


\section{METODOLOGIA}

Este trabalho tem como objetivo apresentar um diagnóstico de estratégias de aprendizagem de IE, no sentido de responder à seguinte questão de pesquisa: quais as estratégias de aprendizagem para desenvolvimento de competências de IE nos estudantes do Ensino Superior? Realizaram-se duas entrevistas focus group (uma a estudantes e outra a Professores) a 10 estudantes de duas instituições de Ensino Superior, uma Pública e uma Privada e a dois Professores de cada uma dessas Instituições. Os estudantes, duas raparigas e os restantes rapazes, dos últimos anos da licenciatura ( $3^{\circ}$ ano), pertencem a 7 licenciaturas diferentes: a) Engenharia Biológica; b) Psicologia; c) Engenharia Química; d) Engenharia de Gestão e Sistemas; e) Engenharia de Sistemas e Informática; f) Turismo; g) Física. Os Professores são Associados (categoria institucional), dois lecionam a área Matemática, um a Física e um a Química, têm em comum, a utilização das metodologias PBL e inverted classroom. Os estudantes têm em comum o facto de serem alunos dos Professores selecionados. Foram solicitadas autorizações por escrito (por um dos autores deste estudo) quer aos coordenadores de Departamentos dos cursos quer aos entrevistados para a realização das audiogravações que se efetuaram nos respetivos Estabelecimentos de Ensino, garantindo-se, obviamente, os anonimatos dos entrevistados e das Instituições.

Na presente pesquisa, após análise de conteúdo (Amado, Costa, \& Crusoé, 2017; Bardin, 2014), das entrevistas audiogravadas resultantes dos dois focus group aos estudantes e aos Professores, procedeu-se à leitura flutuante do conteúdo. Dessa forma, as ideias foram sendo organizadas em unidades de referência, palavras ou frases, textos contidos no material informativo produzido, que correspondiam a ideias claras, objetivas e significativas no contexto da pesquisa. Posteriormente, após a leitura profunda, as unidades de referência foram agrupadas em indicadores, o que posteriormente nos permitiu esclarecer a definição de cada uma das categorias. Como unidade de frequência, usamos a unidade de referência, que foi contada tantas vezes, quantas as presentes no discurso. $O$ trabalho de análise e tratamento dos dados foi realizado com o apoio do software de análise de conteúdo em pesquisa qualitativa, webQDA® (Costa, Moreira, \& Souza, 2019), através de procedimentos abertos, correspondendo a um processo permanente de criação progressiva, no qual a reflexão e análise de dados são triangulados rigorosa e constantemente (Souza, Costa, \& Souza, 2015). 
As entrevistas foram analisadas com o software de análise qualitativa webQDA® e a triangulação dos dados foi realizada entre as entrevistas dos estudantes, as dos Professores e a literatura.

Apesar de o estudo ter categorias a priori, com a ajuda do software webQDA®, incluímos as duas entrevistas nas fontes internas e questionamos as 200 palavras mais frequentes condicionadas a um mínimo de 3 letras, obtendo-se a nuvem de palavras. As palavras mais citadas foram Motivado (101), Ativo (91), Reflexivo (83), Sensorial (82), Empatia (80), Intuitivo (77), Pesquisar (74), Avaliar (67), Sequencial (65), Global (60), Interativo (57), Colaborativo (54), Forte (38), Emotivo (27), Empolgante (25), Compreensivo (23), Respeito (17) e Deduzi (9).

Neste estudo, apresentaremos as dimensões, as categorias e os indicadores, representados no Quadro 1.

Quadro 1. Dimensões, Categorias e Indicadores para o desenvolvimento de IE nos Professores e nos estudantes.

\begin{tabular}{|c|c|c|}
\hline Dimensões & Categorias & Indicadores \\
\hline $\begin{array}{c}\text { Competências } \\
\text { Pessoais }\end{array}$ & $\begin{array}{c}\text { Autoconhecimento } \\
\text { Auto-regulação } \\
\text { Motivação }\end{array}$ & $\begin{array}{c}\text { Ativo, Reflexivo } \\
\text { Forte }\end{array}$ \\
Motivado, Empolgante \\
$\begin{array}{c}\text { Competências } \\
\text { Sociais }\end{array}$ & Empatia \\
Skills Sociais & $\begin{array}{c}\text { Compreensão, } \\
\text { Respeito } \\
\text { Intuitivo, Sensorial, } \\
\text { Interativo, Colaborativo }\end{array}$ \\
\hline $\begin{array}{c}\text { Competências } \\
\text { Académicas }\end{array}$ & Aprendizagem & $\begin{array}{c}\text { Pesquisar, Avaliar, } \\
\text { Deduzir }\end{array}$ \\
\hline
\end{tabular}

As categorias respeitantes às dimensões Competências Sociais e Competências Pessoais são as defendidas por Goleman (1995), a dimensão Competências Académicas e a categoria "Aprendizagem" são emergentes.

Os indicadores de aprendizagem são retirados das palavras mais frequentes. 


\section{TRATAMENTO E ANÁLISE DE RESULTADOS}

Estávamos cientes da ausência de referencial teórico entre IE e metodologias ativas, o que implica, talvez, uma atenção que já de si é enorme e cuidadosa na análise dos dados (Yin, 2015), neste caso em particular, terá de ser redobrada.

Iniciamos o questionamento dos dados para dar resposta à questão de investigação, obtendo as respetivas matrizes de questionamento facilitadas pelo software webQDA®. Questionamos os dados relativamente a correlações entre indicadores que promovem a apresentação de um diagnóstico de indicadores de aprendizagem de IE em estudantes do Ensino Superior.

Fazendo a triangulação dos dados entre os estudantes e os Professores, por categorias, obtivemos a matriz que se traduz na Tabela 2.

Tabela 2. Matriz da triangulação dos dados entre estudantes e Professores.

\begin{tabular}{ccc}
\hline Categorias & $\begin{array}{c}\text { Professores } \\
\text { (Unidade frequência) } \\
\text { (N) }\end{array}$ & $\begin{array}{c}\text { Estudantes } \\
\text { (Unidade frequência) } \\
\text { (N) }\end{array}$ \\
\hline Autoconhecimento & 3 & 10 \\
Auto-regulação & 5 & 6 \\
Motivação & 3 & 7 \\
Empatia & & 8 \\
Skills Sociais & 7 & 11 \\
Aprendizagem & 9 & 7 \\
\hline
\end{tabular}

\subsection{Competências Pessoais}

Analisando a Tabela 2, pode-se verificar que o autoconhecimento é referenciado pelos Professores $(\mathrm{N}=3)$, mas relativamente ao indicador "reflexivo", enquanto os estudantes referem-no ( $\mathrm{N}=10)$ sob o ponto de vista "ativo", sendo que esta divergência tem a ver com o que defendem Erwen, Wenming e Chengxing (2018).

Assim, a metodologia de Inverted Classroom, poderá contribuir para a aprendizagem de skills de IE (Cherniss, Extein, Goleman, \& Weissberg, 2006; Goleman, 1995), uma vez que o estudante poderá aprender em qualquer lugar e em qualquer momento, e assim o tempo na sala de aula é investido, ativamente, na explicação de dúvidas e no aprofundamento de conceitos mais complexos e o Professor tem o papel de proporcionar a reflexividade nos estudantes. 
A auto-regulação é referenciada pelos Professores $(N=5)$ e pelos estudantes $(N=6)$ e o indicador é "Forte". Esta concordância entende-se com o que defende Ribeiro (2008), ou seja o PBL poderá proporcionar skills de IE (Cherniss, Extein, Goleman, \& Weissberg, 2006; Goleman, 1995) no ensino da Engenharia, uma vez que o ciclo PBL torna a aprendizagem do estudante forte nos seus loops (repetições de passos na aprendizagem), de modo a atingir a profundidade esperada sobre o assunto em consideração.

O Professor é um guia que disponibiliza informações sobre o problema antes de cada passo, uma vez que permite que os estudantes desenvolvam elevada performance em contexto de sala de aula (Ó, Almeida, Viana, Sanches, \& Paz, 2019).

A motivação é referenciada por Professores ( $\mathrm{N}=3$ ) como "motivado", e como "empolgante" $(\mathrm{N}=7)$, por parte dos estudantes. Estes resultados, estão concordantes com a metodologia de Inverted Classroom, tal como defendem Fidalgo-Blanco, Sein-Echaluce e Garcia-Peñalvo (2020) e Rossi (2014), podendo proporcionar skills de IE (Cherniss, Extein, Goleman, \& Weissberg, 2006; Goleman, 1995), porque permite aos estudantes e aos Professores trabalharem de forma mais ativa, participativa e cooperativa, havendo mais eficácia e eficiência no rendimento académico, ou seja, "com o mesmo esforço obtém-se melhor resultados, aumentando naturalmente a motivação e o empenho" (Fidalgo-Blanco, SeinEchaluce, \& Garcia-Peñalvo, 2020, p.3).

\subsection{Competências Sociais}

Verificamos que a "empatia" é referenciada pelos Professores $(\mathrm{N}=7)$, no sentido do "respeito" e pelos estudantes ( $N=8$ ), no sentido da "compreensão". Estas skills de IE (Cherniss, Extein, Goleman, \& Weissberg, 2006), podem-se desenvolver nos estudantes do Ensino Superior através da aprendizagem com recurso a metodologias ativas que lhes permitam desenvolver mais altos níveis cognitivos (Almeida, 2019) e de emoções (Franco, 2007; Sousa \& Dias, 2011) na sala de aula. Obrigatoriamente, terá de haver respeito, compreensão e bem-estar geral entre os colegas, ou seja, o respeitar-se e compreender-se a si e aos outros, controlando os pensamentos e os comportamentos (Brakett, \& Salovey, 2006). Estas serão as regras básicas da convivência entre seres humanos em sociedade (Fidalgo-Blanco, Sein-Echaluce, \& Garcia-Peñalvo, 2020), remetendo para os processos que envolvem as competências intra e interpessoais (Mayer, Salovey, \& Caruso, 2004). 
Se passarmos a analisar as skills sociais de IE (Cherniss, Extein, Goleman, \& Weissberg, 2006), em que os Professores ( $N=9$ ) e os estudantes $(N=11)$, de um modo geral, referiram o "intuitivo", o "interativo", o "sensorial" e o "colaborativo", compreende-se que são características que os estudantes obtêm por convivência em grupo. Neste contexto, o PBL, poderá contribuir para skills de IE, devido, à natureza prática, à colaboração interpares, inerentes a esta metodologia, no caso concreto das Engenharias (Ribeiro, 2008). A IE remete para um conjunto de habilidades mentais que permitem perceber e conhecer o significado de padrões das emoções, bem como raciocinar e resolver problemas a partir deles (Mayer \& Salovey, 1997). Nesta linha, a IE permite envolver o estudante na assimilação e aplicação a novas situações do conhecimento curricular, tornando-o agente do seu desenvolvimento pessoal e profissional (Xavier \& Leite, 2019).

Os estudantes desenvolvem uma aprendizagem autónoma, tendo como base processos de aprendizagem ativa recorrendo a uma avaliação diferenciada (Ribeiro, 2008), permitindo-lhes a apropriação de conhecimentos metacognitivos (Sá \& Costa-Lobo, 2019), e o processo de aquisição de conhecimentos é efetivamente ativo e proactivo, autónomo ou colaborativo (Sá, Alves, \& Costa, 2016).

\subsection{Competências Académicas}

Verificamos que o termo "aprendizagem" é referido pelos Professores $(N=6)$ e pelos estudantes ( $\mathrm{N}=7$ ), mas enquanto que os Professores se referem a "deduzir" e "avaliar", os estudantes referem-se a "pesquisar". Estes resultados estão de acordo com Ribeiro (2008), pelo facto de o PBL ser uma metodologia centrada no estudante e, portanto, cabe a eles o rumo da aprendizagem, que pode divergir daqueles previamente planeados pelos Professores.

Os Professores terão a tarefa de levar os estudantes a avaliar passos, metas, e deduzir alguns caminhos que apesar de poderem, aparentemente, não estar corretos, serem um, entre muitos, caminhos possíveis.

Usando a metodologia de Inverted Classroom os estudantes recolhem informações, pesquisando, sempre com a supervisão do Professor, que tem como missão ajudar/apoiar/guiar os estudantes a adquirirem responsabilidade e autonomia progressivas na sua aprendizagem. O Professor assume o papel de facilitador no processo de aprendizagem, cabendo-Ihe acompanhar evolução do estudante em todo o processo (Rossi, 2014), ajudando 
o estudante a desenvolver-se cognitivamente (Leite, Figueiredo, Santos, De Sousa, Sampaio, \& Leal, 2018).

Esta monitorização ou acompanhamento do percurso de aprendizagem dos estudantes por parte dos professores deverá assentar muito no investimento de uma das componentes do conceito de IE, ou seja, "a capacidade de aceder e de gerar sentimentos que facilitem o pensamento" e "a capacidade de regular as emoções para promover o crescimento emocional e intelectual" (Mayer \& Salovey, 1997, p. 15).

Falamos, pois, do desenvolvimento da capacidade, dos alunos gerarem conteúdos positivos na sua mente associados às tarefas de pesquisa.

\section{CONSIDERAÇÕES FINAIS}

As estratégias de aprendizagem para o desenvolvimento de competências de IE nos estudantes do Ensino Superior que mais se evidenciaram neste estudo abrangeram as dimensões de: a) competências pessoais; b) competências sociais; e c) competências académicas.

Podemos concluir que as metodologias ativas, PBL e Inverted Classroom, poderão contribuir para desenvolver skills de IE em estudantes de ensino Superior, dos seguintes modos: a) Professores e estudantes referiram os seguintes indicadores para ser possível adquirir skills de IE de competência pessoal através da aprendizagem a partir de metodologias ativas: ativo, reflexivo, forte, motivado e empolgante; b) Para adquirirem skills de IE de competência social, através da aprendizagem a partir de metodologias ativas, referiram os seguintes indicadores: compreensão, respeito, intuitivo, sensorial, interativo, colaborativo; e c) Por fim, para se adquirir competências académicas, com a aprendizagem através de metodologias ativas, referiram os seguintes indicadores: pesquisar, avaliar e deduzir.

O jogo FLIGBY apresenta-se como uma base de desenvolvimento de uma metodologia de ensino aprendizagem não convencional que pode ser usada em moldes mais latos no sentido de desenvolvimento de competências de IE na educação, dando azo a aplicações não só em unidades curriculares ligadas ao empreendedorismo, mas também no âmbito de outros domínios disciplinares.

As metodologias de aprendizagem ativas, em sala de aula, são pontos de partida para avançar nos processos mais avançados de reflexão, de integração cognitiva, de generalização, de 
reelaboração de novas práticas, de modo a que o Professor possibilite ao estudante desenvolver-se cognitivamente (Leite, 2018; Ó, Almeida, Viana, Sanches, \& Paz, 2019; Xavier \& Leite, 2019) devido à natureza prática, à colaboração intra e interpessoal inerentes a estas metodologias. Neste contexto, surgem um conjunto de habilidades mentais de IE que permitem o raciocínio e a resolução de problemas a partir da perceção e conhecimento de padrões das emoções (Almeida, 2019; Cherniss, Extein, Goleman, \& Weissberg, 2006; Franco, 2007; Mayer, Caruso, \& Salovey, 2000).

A importância das estratégias de aprendizagem a partir de metodologias ativas (Erwen, Wenming, \& Chengxing, 2018; Fidalgo-Blanco, Sein-Echaluce, \& Garcia-Peñalvo, 2020; Rossi, 2014; Ribeiro, 2008; Sá, Alves, \& Costa, 2016) para o desenvolvimento das competências de IE nos estudantes de Ensino Superior, são extremamente importantes para a aprendizagem, constituindo-se em aprendizagens a aplicar e rentabilizar no sentido do sucesso escolar, e também do sucesso profissional.

Em trabalhos futuros, seria útil propor uma grelha de diagnóstico de estratégias de aprendizagem com metodologias ativas e investigar o impacto da IE na aprendizagem em determinadas unidades curriculares. Seria importante comparar os ganhos de aprendizagem e o sucesso de desempenho de IE dos estudantes: a) que aprendem com abordagens de metodologias ativas; b) que aprendem apenas com outras abordagens académicas; e c) bem como avaliar o impacto de variáveis como género, idade e curso no desenvolvimento de tal aprendizagem.

\section{REFERÊNCIAS}

Alexiou, A., Schippers, M., \& Oshri, I. (2012). Positive Psychology and Digital Games: The Role of Emotions and Psychological Flow in Serious Games Development. Psychology, 3(12), 1243-1247.

Almeida, F. (2019). Adoption of a serious game in the Developing of Emotional Intelligence Skills. European Journal of Investigation in Health, Psychology and Education, 10(1), 30-43. doi: https://doi.org/10.3390/ ejihpe10010004.

Almeida, F., \& Buzády, Z. (2019). Learning Entrepreneurship in Higher Education Through Flow Theory and FLIGBY Game. International Journal of Virtual and Personal Learning Environments, 9(1), 1-15. doi:10.1016/j.entcom.2014.07.003.

Amado, J., Costa, A., \& Crusoé, N. (2017). A técnica de análise de conteúdo. In: Manual de Investigação qualitativa em educação. (2 $2^{\text {nd }}$ ed.).(pp. 301-355). Coimbra Universty Press, Coimbra. doi: http://dx.doi.org/10.14195/978-989-26-0879-2.

Bardin, L. (2014). Análise de Conteúdo. (5 $5^{\text {th }}$ ed). Lisboa: Edições 70.

Berbel, N. (2011). As metodologias ativas e a promoção da autonomia de estudantes. Semina: Ciências Sociais e Humanas, Londrina, 32(1), 25-40. doi: http://dx.doi.org/10.5433/1679- 0383.2011v32n1p25. 
Brackett, M. A., \& Salovey, P. (2006). Measuring emotional intelligence with the Mayer-Salovery-Caruso Emotional Intelligence Test (MSCEIT). Psicothema, 18, 34-41.

Câmpeanu-Sonea, E., Sonea, A., \& Bresfelean, V. P. (2013). The Role of Emotional Intelligence in Labour Market Orientation and Career Development. Management Dynamics in the Knowledge Economy, 1(1), 127-142.

Cherniss, G., Extein, M., Goleman, D., \& Weissberg, R. P. (2006). Emotional Intelligence: What does the Research Really Indicate? [Comment/Reply]. Educational Psychologist, 41(4), 239-245. doi: http://dx.doi.org/10.1207/s15326985ep4104_4

Csikszentmihalyi, M. (2003). Good Business: Leadership, Flow and the Making of Meaning. New York, NY: Penguim Books.

Costa, A. P., Moreira, A., \& Souza, F. (2019). webQDA (version 3.1) - Qualitative Data Analysis. Aveiro University and MicrolO: Aveiro.

Erwen, Z., Wenming, Z., \& Chengxing, J. (2018). SPOC-based Flipped Classroom of College English: Construction of an Effective Learning Model. iJET, 13(1), 37-45. doi: 10.3991/ijet.v13i01.7513.

Fidalgo-Blanco, Á., Sein-Echaluce, M. L., \& García-Peñalvo, F. J. (2020). Ventajas reales en la aplicación del método de Aula Invertida-Flipped Classroom. Zenodo, 1(1), 1-8. doi: http://doi.org/10.5281/zenodo.3610578.

Franco, M. (2007). Inteligência humana. In Almeida, L., \& Candeias, A. (Coords.), vol. 1. Inteligência emocional: modelos, instrumentos de avaliação e limites. (pp. 73-96). Coimbra: Quarteto.

Goleman, D. (1995). Emotional Intelligence: Why it Can Matter More Than IQ. Bantam Books: New York.

Leite, C., Figueiredo, C., Santos, C., De Sousa, R., Sampaio, M., \& Leal, R. (2018). Ensino Superior: Desafios e Futuros. Porto: CIIE

Mayer, J. D., Caruso, D. R., \& Salovey, P. (2000). Emotional Intelligence Meets Traditional Standars for na Intelligence. Intelligence, 27(4), 267-298. doi: http://dx.doi.org/10.1016/S0160-2896(99)00016-1.

Mayer, J. D., \& Salovey, P. (1997). What is Emotional Intelligence? In P. Salovey, \& D. Sluyter (Eds.), Emotional development and emotional intelligence. Educational Implications. (pp. 3-31). New York: Basic Books.

Mayer, J. D., Salovey, P., \& Caruso, D. R. (2004). Emotional Intelligence: Theory, Findings and Implications. Psychol. Inq., 15, 197-215.

Ó, J., Almeida, M., Viana, J., Sanches, T., \& Paz, A. (2019). Tendências recentes da investigação internacional sobre pedagogia do ensino superior: uma revisão da literatura. Revista Lusófona de Educação, 45, $205-221$. doi: 10.24140/issn.1645-7250.rle45.14.

Ribeiro, L. (2008). Aprendizagem Baseada em Problemas (PBL) na Educação em Engenharia. Revista de Ensino de Engenharia, 27(2), 23-32.

Rossi, R. D. (2014). Improving Student Engagement in Organic Chemistry using the Inverted Classroom Model. ACS CHED CCCe: Spring. 
Sá, S., Alves, P., \& Costa, A. (2016). Methods of Teaching Centered on Learning and Formative assessment in Higher Education. The Qualitative Report, 21(13), 54-62.

Sá, S., \& Costa-Lobo, C. (2019). Evaluate the Pedagogical Practice of the Teachers of Higher Education: A Proposal. REICE. Revista Iberoamericana sobre Calidad, Eficacia y Cambio en Educación, 17(1), 61-76. doi: https://doi.org/10.15366/reice2019.17.1.004.

Sahoo, F., \& Sahu, R. (2009). The Role of Flow Experience in Human Happiness. Journal of the Indian Academy of Applied Psychology, 35, 40-47.

Sousa, A. B., \& Dias, J. H. (2011). Inteligência Emocional e Desempenho Académico em Estudantes do Ensino Superior. Interações, 21, 95-124.

Souza, D., Costa, A., \& Souza, F. (2015). Desafio e inovação do estudo de caso com apoio das tecnologias. In: Investigação Qualitativa: Inovação, Dilemas e Desafios. (pp. 143-162). Ludomedia: Oliveira de Azeméis, Aveiro.

Xavier, A., \& Leite, C. (2019). Mapeamento da Formação pedagógica dos docentes universitários nas Universidades Públicas Portuguesas. Revista Lusófona de Educação, 45, 109-123. doi: 10.24140/issn.16457250.rle45.08.

Yin, R. K. (2015). Case studies research: design and methods. ( $5^{\text {th }}$ ed.). Thousands Oaks: Sage.

Zumeta, L., Basabe, N., Wlodarczyk, A., Bobowik, M., \& Páez, D. (2016). Shared flow and positive collective gatherings. Anales de Psicologia, 32(3), 717-727. 